CrossMark \& click for updates

Cite this: Phys. Chem. Chem. Phys., $2015,17,27873$

Received 26th August 2015, Accepted 14th September 2015

DOI: $10.1039 / c 5 c p 05088 j$

www.rsc.org/pccp

\section{Acidity of two-dimensional zeolites $\dagger$}

\begin{abstract}
Marcin Rybicki and Joachim Sauer*
Hybrid quantum mechanics:molecular mechanics (QM/MM) calculations of absolute deprotonation energies are performed with periodic boundary conditions for Brønsted sites of aluminosilicate bilayers with various $\mathrm{Al} / \mathrm{Si}$ ratios (two-dimensional zeolite). The supercell method is applied and density functional theory is used. Much lower values are obtained (1042, 1069 and $1091 \mathrm{~kJ} \mathrm{~mol}^{-1}$ for Al/Si $=1 / 63$, $1 / 7$ and $1 / 3$, respectively) than those for bulk zeolites $\left(1233 \mathrm{~kJ} \mathrm{~mol}^{-1}\right.$ for $\mathrm{H}$-chabazite with $\left.\mathrm{Al} / \mathrm{Si}=1 / 11\right)$. We ascribe the much lower deprotonation energy to the smaller effective dielectric constant (1.6-1.9) of an ultra-thin dielectric in a vacuum compared to that of the corresponding bulk systems (3.0 for $\mathrm{H}$-chabazite), which leads to a better stabilization of the charge created upon deprotonation.
\end{abstract}

\section{Introduction}

Zeolites are crystalline porous aluminosilicates which are naturally occurring or synthesized. They are widely used as catalysts in industrial processes such as catalytic cracking of crude oil, methanol to gasoline conversion and olefin oligomerization. ${ }^{1,2}$ The catalytic activity of proton forms of zeolites originates from bridging $\mathrm{Al}-\mathrm{O}(\mathrm{H})-\mathrm{Si}$ groups which represent Brønsted acid sites. ${ }^{3,4}$

In recent years a particular class of materials, two-dimensional zeolites, has attracted interest. ${ }^{5}$ They exist either as nanosheets, as thin as single unit cells of, e.g. MFI, ${ }^{6,7}$ or as ultra-thin model systems grown under UHV conditions on metal substrates. ${ }^{8,9}$ The latter were prepared by the deposition of $\mathrm{O}_{2}, \mathrm{Si}$ and $\mathrm{Al}$ on the $\mathrm{Ru}(0001)$ surface and subsequent adsorption and desorption of water. ${ }^{8,9}$ They consist of an aluminosilicate bilayer with a two-dimensional arrangement of hexagonal prisms and contain surface Brønsted sites. ${ }^{8}$ Thus, they may be considered as two-dimensional zeolites $(\mathrm{H}-2 \mathrm{dH})$ with the flat surface corresponding to the infinite pore size. ${ }^{9}$ The advantage of the $\mathrm{H}-2 \mathrm{dH}$ thin films is that their properties can be analysed using surface science techniques.

Adsorption of the weak bases $\mathrm{CO}$ and $\mathrm{C}_{2} \mathrm{H}_{2}$ on the acidic site of the $\mathrm{H}-2 \mathrm{dH}$ zeolite causes a red shift of the $\mathrm{O}-\mathrm{H}$ stretching band that is considerably higher than the shift observed for the bulk $\mathrm{H}$-SSZ-13 zeolite, the protonated form of $\mathrm{H}$-chabazite (H-CHA) ${ }^{8,9}$ This suggests that the two-dimensional (2D) zeolite structures are considerably more acidic than the three-dimensional (3D) ones.

Institut für Chemie, Humboldt-Universität zu Berlin, Unter den Linden 6,

10099 Berlin, Germany.E-mail: js@chemie.hu-berlin.de

$\dagger$ Electronic supplementary information (ESI) available: Unit cell parameters, geometric parameters, figures of cluster models, and additional results for deprotonation energies as well as $x, y$, and $z$ coordinates for selected structures. See DOI: $10.1039 / \mathrm{c} 5 \mathrm{cp} 05088 \mathrm{j}$
The $\mathrm{OH}$ frequency shift upon adsorption of probe molecules ${ }^{10}$ is only one of the different measures of acidity strength. ${ }^{11-13}$ It is a reactivity parameter that assumes an "early" transition state. In contrast, adsorption of strong bases like ammonia or pyridine that have also been studied for $\mathrm{H}-2 \mathrm{dH}$ and $\mathrm{H}-\mathrm{CHA}$ catalysts $^{9}$ implies a late transition state with the proton being transferred to the reaction partner. However, the heat of ammonia adsorption depends on two factors, the intrinsic acidity of the Brønsted site (which is larger for the $2 \mathrm{D}$ zeolite) and the van der Waals interaction between the ammonium ion formed and the surface. The latter is larger for the curved surface of the porous material which is due to a better fit. A parameter that describes the intrinsic acidity without any interaction between the base molecule and the surface is the deprotonation energy (or the proton affinity of the deprotonated Brønsted site). ${ }^{4}$

The energy of adsorption, $\Delta E_{\mathrm{ads}}$, for the formation of an interacting ion pair

$$
\mathrm{Z}-\mathrm{OH}+\mathrm{B} \rightarrow \mathrm{Z}^{-} \mathrm{O}^{-} \mathrm{HB}^{+}
$$

can be decomposed into the energy of the interaction, $\Delta E_{\mathrm{IP}}$, between the protonated species and the deprotonated surface site,

$$
\mathrm{Z}-\mathrm{O}^{-}+\mathrm{HB}^{+} \rightarrow \mathrm{Z}^{-} \mathrm{O}^{-} \mathrm{HB}^{+}
$$

the deprotonation energy, $\Delta E_{\mathrm{DP}}$, of the Brønsted site,

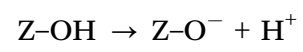

and the proton affinity, $\Delta E_{\mathrm{PA}}$, of the base molecule

$$
\mathrm{B}+\mathrm{H}^{+} \rightarrow \mathrm{HB}^{+}
$$

Hence,

$$
\Delta E_{\mathrm{ads}}=\Delta E_{\mathrm{IP}}+\Delta E_{\mathrm{DP}}+\Delta E_{\mathrm{PA}}
$$


For molecules in the gas phase measuring the deprotonation energy (or the proton affinity) is a well-established technique. Mass spectrometry yields proton transfer equilibrium constants that are converted into gas phase acidity and gas phase basicity scales, e.g., ref. 14. For surface hydroxyl groups, however, quantum chemical calculations are the only source.,11,15,16

The aim of this work is to determine the intrinsic acidity of $\mathrm{H}-2 \mathrm{dH}$ zeolites by performing hybrid QM/MM calculations of the deprotonation energy, and to compare it with that of $\mathrm{H}-\mathrm{CHA}$ as an example of bulk zeolites. We also investigate the influence of the $\mathrm{Al} / \mathrm{Si}$ ratio on the acidity of the $\mathrm{H}-2 \mathrm{dH}$ system and we analyse the structure factors determining the deprotonation energies.

\section{Methods}

\subsection{Periodicity and deprotonation energies}

Zeolites are crystalline materials and theoretical investigations of their properties have to take into account the periodicity of the system. The deprotonation energy of a zeolite $\left(\Delta E_{\mathrm{DP}}\right)$ is defined as the energy difference between the deprotonated $\left(\mathrm{Z}-\mathrm{O}^{-}\right)$and the protonated $(\mathrm{Z}-\mathrm{OH})$ zeolite:

$$
\Delta E_{\mathrm{DP}}=E_{\mathrm{ZO}^{-}}-E_{\mathrm{ZOH}}=\Delta E_{\mathrm{DP}}^{\mathrm{v}}+E_{\mathrm{rel}}
$$

Using the energy of the deprotonated system "at the structure of" the parent system, $E_{\mathrm{ZO}^{-} / / \mathrm{ZOH}}$, it may be decomposed into a "vertical" deprotonation energy, $\Delta E_{\mathrm{DP}}^{\mathrm{v}}$, and a relaxation energy, $E_{\text {rel }}$,

$$
\begin{gathered}
E_{\mathrm{rel}}=E_{\mathrm{ZO}^{-}}-E_{\mathrm{ZO}^{-} / / \mathrm{ZOH}} \\
\Delta E_{\mathrm{DP}}^{\mathrm{v}}=E_{\mathrm{ZO}^{-} / / \mathrm{ZOH}}-E_{\mathrm{ZOH}}
\end{gathered}
$$

Applying periodic boundary conditions to the deprotonation process implies that a negative charge is created in every unit cell. This causes divergence of the electrostatic energy for the deprotonated system, $\mathrm{ZO}^{-}$. It is also an unrealistic model because in the catalytic process we do not expect release of a proton in every unit cell at the same time. The divergence of the electrostatic energy can be eliminated by adding a uniform background charge to the deprotonated unit cell. The spurious interaction between the charged defects can be reduced using the supercell method or approximately eliminated using the method proposed by Leslie and Gillan (LG method). ${ }^{17}$

2.1.1 The supercell approach. This is the simplest, but computationally most demanding method. We build a series of supercells by multiplying the original unit cell $N$ times in every direction and calculate the deprotonation energy for each supercell. We remove only one proton per supercell. This way, the distance between charged defects increases, and the artificial anion-anion interaction decreases. The deprotonation energy of the zeolite becomes a function of $N$. In the limit of infinitely large $N$, its value, $\Delta E_{\mathrm{DP}}^{\infty}$, is interpreted as the deprotonation energy needed to remove the proton from an isolated Brønsted site. In the $N \times N \times N$ supercell, the cubic root of the supercell volume, $\sqrt[3]{V}$, can be defined as the average distance between the charged defect and its nearest periodic images, $\left\langle r_{--}\right\rangle$. The deprotonation energy of the system can be extrapolated using the following equation:

$$
\Delta E_{\mathrm{DP}}(N)=\Delta E_{\mathrm{DP}}^{\infty}+\frac{A}{\left\langle r_{--}\right\rangle}+\frac{B}{\left\langle r_{--}\right\rangle^{3}}=\Delta E_{\mathrm{DP}}^{\infty}+E_{\mathrm{corr}}
$$

where $\Delta E_{\mathrm{DP}}(N)$ is the deprotonation energy for the $N \times N \times N$ supercell, and $A$ and $B$ are fitting parameters. The $1 / r$ and $1 / r^{3}$ terms of the total correction, $E_{\text {corr }}$, correspond to charge-charge and dipole-dipole interactions, respectively, and the parameters $A$ and $B$ reflect the screening properties of the material.

2.1.2 Aperiodic correction. The method proposed by Leslie and Gillan $^{17}$ relies on the macroscopic approximation. It assumes that the difference between the energy per unit cell for the periodic defect and for the isolated defect is equal to the interaction of a periodic array of point charges, $E_{--}$, immersed in a dielectric medium with the dielectric constant $\varepsilon$ of the system without defects. Therefore, the deprotonation energy can be expressed as:

$$
\Delta E_{\mathrm{DP}}^{\mathrm{LG}}=\Delta E_{\mathrm{DP}}-\frac{E_{--}}{\varepsilon(\mathrm{LG})}
$$

In practice, $E_{--}$is the energy of the point charge (usually $\mathrm{H}^{+}$) placed in the unit cell of the investigated system immersed in the compensating background charge, whereas $\varepsilon(\mathrm{LG})$ is $1 / 3$ of the trace of the static dielectric tensor of the system. Comparison of eqn (8) and (9) shows that, if both correction methods are correct, the relationship:

$$
\frac{E_{--}}{\varepsilon}=\frac{A}{\left\langle r_{--}\right\rangle}+\frac{B}{\left\langle r_{--}\right\rangle^{3}}
$$

holds, from which we can calculate an effective dielectric constant $\varepsilon_{\text {eff }}(\mathrm{SC})$ from given $A$ and $B$ parameters. The LeslieGillan approach is valid if the original unit cell is big enough to encapsulate the structural deformation induced by the charged defect and the dielectric constant of the material is isotropic. Both conditions are usually fulfilled for bulk zeolites, and the Leslie-Gillan method of aperiodic correction is the method of choice for these materials.

\subsection{Embedded cluster QM/MM calculations}

To calculate the deprotonation energy of zeolites, we used a mechanical embedding scheme, ${ }^{18,19}$ which partitions the system (S) into the inner region (I) and the outer region (O). The dangling bonds of the inner part are saturated with hydrogen link atoms, and the inner part together with the link atoms forms the cluster (C). The total energy $E(\mathrm{~S})$ and the forces $\vec{F}_{\alpha}(\mathrm{S})$ acting on the atoms are obtained as follows: ${ }^{18,19}$

$$
\begin{gathered}
E(\mathrm{~S})=E_{\mathrm{QM}}(\mathrm{C})+E_{\mathrm{MM}}(\mathrm{S})-E_{\mathrm{MM}}(\mathrm{C}) \\
\vec{F}_{\alpha}(\mathrm{S})=\vec{F}_{\alpha, \mathrm{QM}}(\mathrm{C})+\vec{F}_{\alpha, \mathrm{MM}}(\mathrm{S})-\vec{F}_{\alpha, \mathrm{MM}}(\mathrm{C}) \quad \alpha \in \mathrm{I} \\
\vec{F}_{\beta}(\mathrm{S})=\vec{F}_{\beta, \mathrm{MM}}(\mathrm{S}) \quad \beta \in \mathrm{O}
\end{gathered}
$$

To the quantum mechanical results for the cluster, $E_{\mathrm{QM}}(\mathrm{C})$ and $\vec{F}_{\alpha, \mathrm{QM}}(\mathrm{C})$, the results of the periodic description of the lattice by the force field, $E_{\mathrm{MM}}(\mathrm{S})$ and $\vec{F}_{\alpha, \mathrm{MM}}(\mathrm{S})$, are added. The third contribution $E_{\mathrm{MM}}(\mathrm{C})$ and $\vec{F}_{\alpha, \mathrm{MM}}(\mathrm{C})$ eliminates approximately 
the double counting of the contributions coming from atoms in the inner region and artificial contributions from link atoms. For an atom $\alpha$ of the inner region, all three terms contribute to the force acting on it. For an atom $\beta$ of the outer region, the force is obtained from the force field alone. A link atom is not moved according to the force acting on it. It is instead fixed on the bond which it terminates. ${ }^{19,20}$

If we apply the QM-Pot embedding scheme (eqn (11a)) to calculate the zeolite deprotonation energy and introduce the notation $\mathrm{CH}, \mathrm{SH}, \mathrm{C}^{-}$and $\mathrm{S}^{-}$, for the cluster $\mathrm{C}$ and the total system $\mathrm{S}$ of the protonated and deprotonated form, respectively, we obtain:

$$
\Delta E_{\mathrm{DP}}=\Delta E_{\mathrm{DP}(\mathrm{QM})}+\Delta E_{\mathrm{DP}(\mathrm{MM})}
$$

with:

$$
\begin{gathered}
\Delta E_{\mathrm{DP}(\mathrm{QM})}=E_{\mathrm{QM}}\left(\mathrm{C}^{-}\right)-E_{\mathrm{QM}}(\mathrm{CH}) \\
\Delta E_{\mathrm{DP}(\mathrm{MM})}=E_{\mathrm{MM}}\left(\mathrm{S}^{-}\right)-E_{\mathrm{MM}}\left(\mathrm{C}^{-}\right)-\left[E_{\mathrm{MM}}(\mathrm{SH})-E_{\mathrm{MM}}(\mathrm{CH})\right]
\end{gathered}
$$

If the cluster is large enough, the structural distortion upon deprotonation decays within the cluster region and the $\Delta E_{\mathrm{DP}(\mathrm{MM})}$ term consists only of electrostatic interactions, i.e. long-range contributions $\Delta E_{\mathrm{LR}}{ }^{18}$ Hence,

$$
\Delta E_{\mathrm{DP}}=\Delta E_{\mathrm{DP}(\mathrm{QM})}+\Delta E_{\mathrm{LR}}
$$

Effectively, the deprotonation energy of the whole system is the sum of the deprotonation energy of a cluster calculated at the QM level and the long-range term representing the electrostatic interaction between the inner and the outer part of the system. This long range term is described at the MM level. Thus, the choice of an appropriate force field is crucial for the success of $\mathrm{QM} / \mathrm{MM}$ calculations. To check if the applied embedding scheme works correctly, the convergence of the deprotonation energy with respect to the cluster size has to be investigated.

For the Leslie-Gillan method, with eqn (9) the total longrange correction, $\Delta E_{\mathrm{LR}}^{\infty}$, is given by

$$
\Delta E_{\mathrm{LR}}^{\infty}=\Delta E_{\mathrm{DP}}^{\mathrm{LG}}-\Delta E_{\mathrm{DP}(\mathrm{QM})}=\Delta E_{\mathrm{LR}}-\frac{E_{--}}{\varepsilon(\mathrm{LG})}
$$

whereas for the supercell method it is obtained as

$$
\Delta E_{\mathrm{LR}}^{\infty}=\Delta E_{\mathrm{DP}}^{\infty}-\Delta E_{\mathrm{DP}(\mathrm{QM})}
$$

\subsection{Details of QM/MM calculations}

We used the QMPOT package, ${ }^{19,20}$ with DFT for the QM part and the DFT-parametrized polarizable shell model potential of Sierka and Sauer ${ }^{21}$ for the MM part. The DFT calculations of energies and forces were done using the TURBOMOLE ${ }^{22}$ package with the B3LYP functional and the TZVP basis set named "def2" in the TURBOMOLE library. ${ }^{23,24}$ The latter includes the following contracted functions: $\mathrm{H}$ : $(5 \mathrm{~s} 1 \mathrm{p}) /[3 \mathrm{~s} 1 \mathrm{p}]$, O: (11s6p2d1f)/[5s3p2d1f], and Si/Al: (14s9p3d1f)/[5s5p2d1f]. The choice of functional is based on previous experiences, ${ }^{21,25}$ and motivated by the fact that deprotonation energies of small aluminosilicate clusters calculated with the B3LYP functional are in very good agreement with the $\operatorname{CCSD}(\mathrm{T})$ results (see Table S1 in the ESI $\dagger$ ). For the shell model MM calculations, we used the GULP code, ${ }^{26}$ with parameters derived from B3LYP cluster calculations. $^{21}$ It was shown ${ }^{21}$ that for this combination of QM and $\mathrm{MM}$ methods deprotonation energies of zeolites converge to a constant value with increasing cluster size. This indicates that the polarizable shell-model force field describes the electrostatic interactions as accurately as the B3LYP functional.

\section{Results}

\subsection{H-CHA}

The H-CHA zeolite is chosen as a reference system because it consists of hexagonal prisms as secondary building units like the $\mathrm{H}-2 \mathrm{dH}$ zeolite (Fig. 1). ${ }^{9}$ All tetrahedral atoms of $\mathrm{H}-\mathrm{CHA}$ are crystallographically equivalent (Fig. 1) and there are only four distinct oxygen positions. ${ }^{28}$ The energies of structures with the proton attached to different oxygen positions are similar, ${ }^{25}$ therefore we calculate deprotonation energies for the lowest energy structure only, i.e. with the proton attached to the $\mathrm{O} 1$ oxygen. We used a unit cell consisting of 37 atoms, ${ }^{20,21}$ with HAlSi ${ }_{11} \mathrm{O}_{24}$ composition and cell parameters optimised using the shell model potential (see Table S2 in the ESI $\dagger$ ). These unit

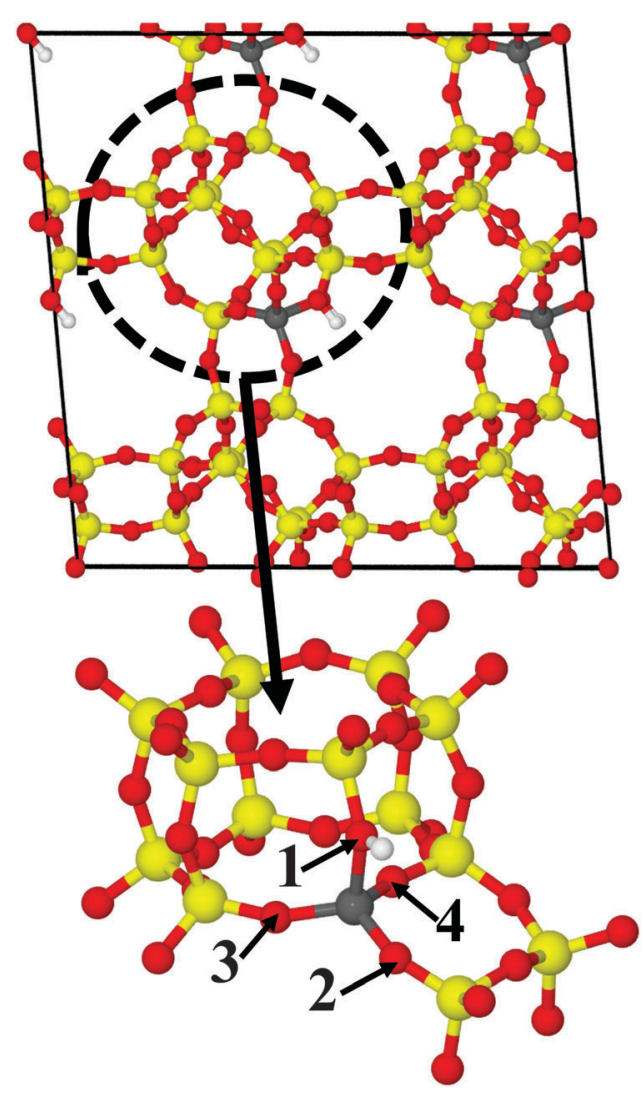

Fig. 1 Optimized $2 \times 2 \times 2$ unit cell of $\mathrm{H}-\mathrm{CHA}$ zeolite together with the numbering of oxygen atoms. The following colour scheme is adopted: silicon, oxygen, aluminium and hydrogen are depicted in yellow, red, grey and white, respectively. The pictures of molecular models were generated using the Jmol program. ${ }^{27}$ 
cell parameters were fixed during subsequent structure optimisation of protonated and deprotonated forms of the zeolite, for which we used the following convergence criteria: a maximum energy change of $1 \times 10^{-5} \mathrm{eV}$, a maximum gradient component of $1 \times 10^{-2} \mathrm{eV}^{-1}$, and a maximum displacement component of $1 \times 10^{-3} \AA$. Cluster models of increasing size were generated automatically by selecting only those tetrahedral sites which were within a defined distance from the $\mathrm{O} 1$ site.

The QM clusters were always terminated with $\mathrm{OH}$ groups with a fixed $\mathrm{OH}$ distance of $96.1 \mathrm{pm}$ (Fig. 2). This is an equilibrium distance that we obtained by free cluster optimization of similar cluster models.

To compare Leslie-Gillan deprotonation energies (eqn (9)) with those obtained using the supercell method we performed calculations for an embedded $8 \mathrm{~T}_{12 \mathrm{OH}}$ cluster model (eight tetrahedral sites terminated by $12 \mathrm{OH}$ groups) with increasing supercell size from $2 \times 2 \times 2$ to $4 \times 4 \times 4$. Fig. 3 and Table 1 show that the corrected deprotonation energies of both methods, $\Delta E_{\mathrm{DP}}^{\mathrm{LG}}$ and $\Delta E_{\mathrm{DP}}^{\infty}$, agree within $0.6 \mathrm{~kJ} \mathrm{~mol}^{-1}$.

The increase of $\Delta E_{\mathrm{DP}(\mathrm{QM} / \mathrm{MM})}$ with increasing cell size is perfectly compensated by the decrease of the aperiodic correction, $E_{-} / \varepsilon(\mathrm{LG})$ (Leslie-Gillan method) or by the decrease of the $A /\left\langle r_{--}\right\rangle,+B /\left\langle r_{--}\right\rangle^{3}$ correction term (super-cell method) so that the $\Delta E_{\mathrm{DP}}^{\mathrm{LG}}$ and $\Delta E_{\mathrm{DP}}^{\infty}$ values, respectively, are perfectly converged already for the smallest $2 \times 2 \times 2$ cell.

The dielectric constants calculated from the fitted parameters $A$ and $B$ (Fig. 3) and the average dielectric constants calculated for the LG correction method from the shell-model

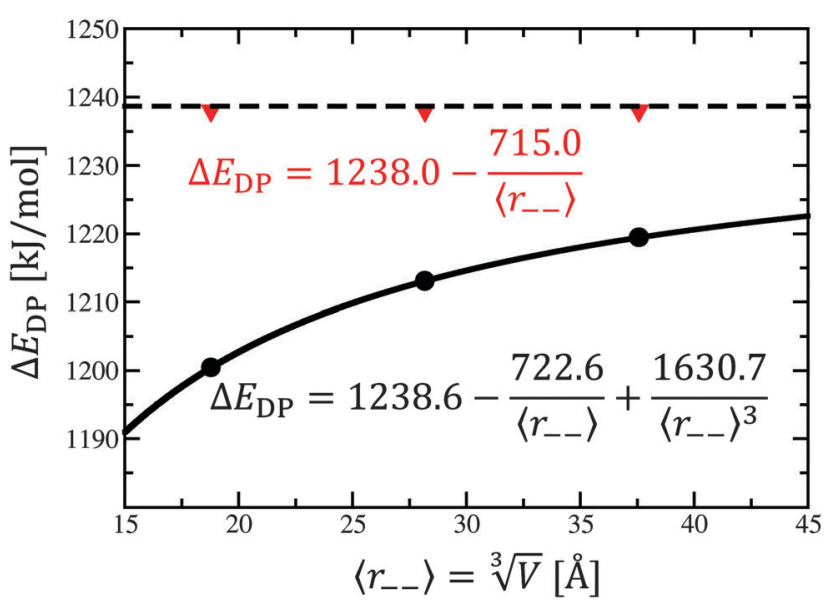

Fig. 3 Uncorrected deprotonation energies of the $8 \mathrm{~T}_{12 \mathrm{OH}}$ cluster model of $\mathrm{H}-\mathrm{CHA}$ embedded in $\mathrm{N} \times \mathrm{N} \times \mathrm{N}$ supercells (black dots). The solid line is the fitting curve, eqn (8). Deprotonation energies corrected using the LG method (red triangles) as a function of the cubic root of the unit cell volume and extrapolated deprotonation energy (dashed line).

potential are virtually identical. The variation of $\varepsilon(\mathrm{LG})$ with the cell size is less than $2 \%$, with the cluster size (Table 2) being slightly larger, $5 \%$.

The QM part of the deprotonation energy is almost independent of the supercell size. This shows that the cluster structure is not affected by the unit cell size and structural distortions do not extend beyond the $2 \times 2 \times 2$ supercell. In absolute terms, the

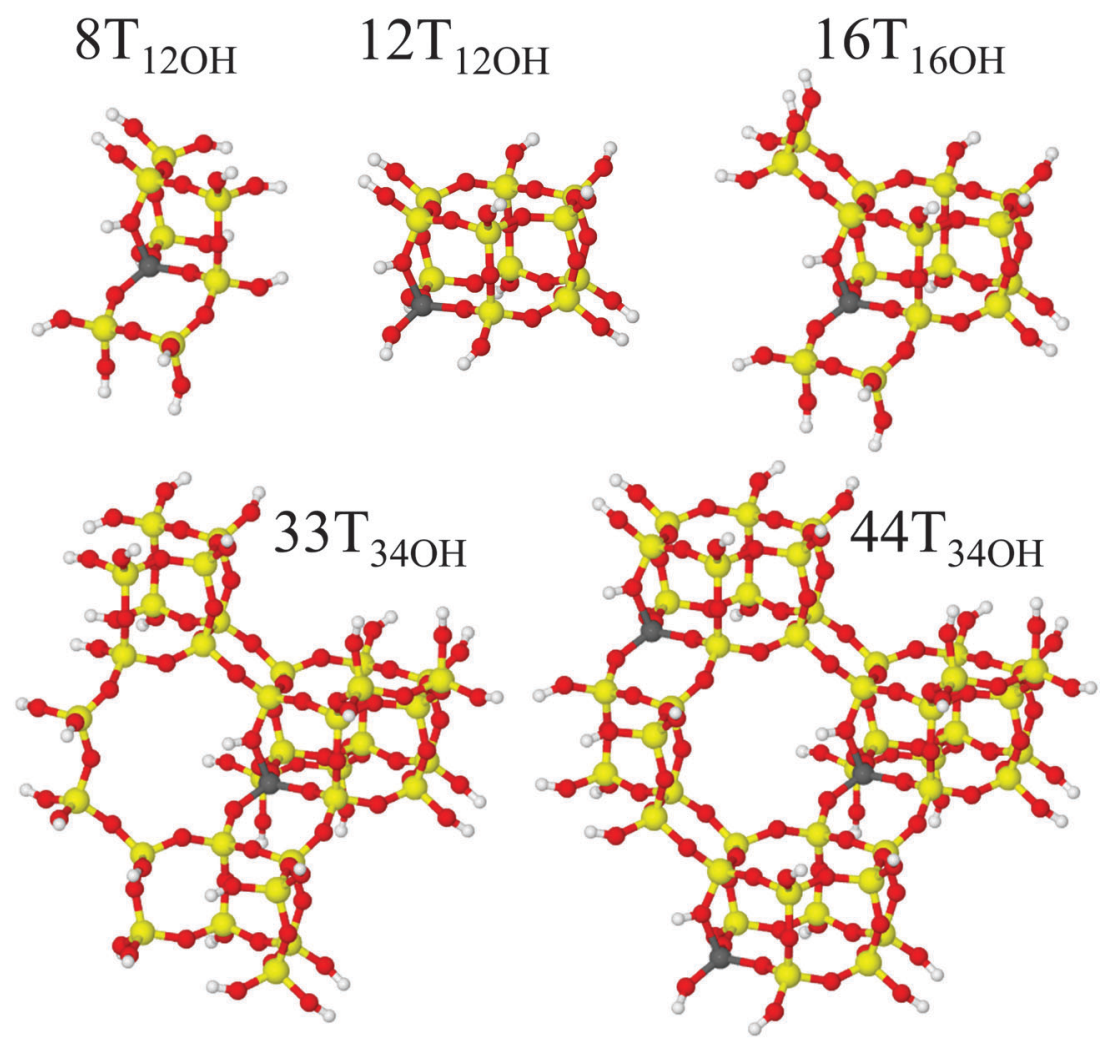

Fig. 2 Cluster models applied in QM/MM calculations of the H-CHA deprotonation energy. 
Table 1 Deprotonation energies $\left(\mathrm{kJ} \mathrm{mol}^{-1}\right)$ and their components for the $8 \mathrm{~T}_{12 \mathrm{OH}}$ cluster model of $\mathrm{H}-\mathrm{CHA}$ embedded in different $\mathrm{N} \times \mathrm{N} \times \mathrm{N}$ supercells: $\Delta E_{\mathrm{DP}}^{\mathrm{LG}}$ and $\Delta E_{\mathrm{DP}}^{\infty}$ - deprotonation energies calculated using the Leslie-Gillan (LG) and supercell (SC) methods, respectively; $\Delta E_{\mathrm{DP}(\mathrm{QM} /}$ $\mathrm{MM})$ and $\Delta E_{\mathrm{DP}(\mathrm{QM})}$ - (uncorrected) $\mathrm{QM} / \mathrm{MM}$ deprotonation energy and its QM part, respectively. Dielectric constants calculated as $1 / 3$ of the trace of the dielectric constant matrix at the MM level (shell-model potential) (LG), and calculated from eqn (10) using the fitted parameters $A$ and $B$, and $\varepsilon_{\text {eff }}(\mathrm{SC})$

\begin{tabular}{lccc}
\hline Unit cell & $2 \times 2 \times 2$ & $3 \times 3 \times 3$ & $4 \times 4 \times 4$ \\
\hline$\Delta E_{\mathrm{DP}}^{\mathrm{LG}}$ & 1238.0 & 1238.0 & 1238.0 \\
$E_{--} / \varepsilon(\mathrm{LG})^{a}$ & -37.6 & -24.9 & -18.6 \\
$\Delta E_{\mathrm{DP}(\mathrm{QM} / \mathrm{MM})}$ & 1200.4 & 1213.1 & 1219.4 \\
$\Delta E_{\mathrm{DP}(\mathrm{QM})}$ & 1291.7 & 1292.4 & 1292.3 \\
$\Delta E_{\mathrm{DP}}^{\infty}$ & 1238.6 & 1238.6 & 1238.6 \\
$A /\left\langle r_{--}\right\rangle^{b}$ & -38.42 & -25.61 & -19.22 \\
$B /\left\langle r_{--}\right\rangle^{3 b}$ & 0.22 & 0.07 & 0.02 \\
$\varepsilon(\mathrm{LG})$ & 2.79 & 2.81 & 2.83 \\
$\varepsilon_{\text {eff }}(\mathrm{SC})$ & 2.75 & 2.74 & \\
${ }^{a}$ Eqn $(9) .{ }^{b}$ Eqn (10). & & & \\
\hline
\end{tabular}

charge-charge interaction, $A /\left\langle r_{--}\right\rangle$, is more than two orders of magnitude larger than the dipole-dipole aperiodic correction term, $B /\left\langle r_{--}\right\rangle^{3}$ (Table 1). This supports the assumption of Leslie and Gillan that the aperiodic correction is given by the chargecharge interaction. We use the $A /\left\langle r_{--}\right\rangle$term only to correct all subsequent deprotonation energies of the H-CHA zeolite.

The results obtained with embedded clusters of increasing size (Table 2) show that the corrected deprotonation energies of the $\mathrm{H}$-CHA zeolite converge to $1233 \pm 5 \mathrm{~kJ} \mathrm{~mol}^{-1}$. This energy is in very good agreement with the value of $1231 \mathrm{~kJ} \mathrm{~mol}^{-1}$, determined using the $\mathrm{QM} / \mathrm{MM}$ methodology with a smaller basis set, ${ }^{29}$ and with the value of $1235 \mathrm{~kJ} \mathrm{~mol}^{-1}$ obtained from the planewave DFT calculation with the BLYP functional (corrected for the systematic error of the functional). ${ }^{29}$ A periodic planewave DFT calculation with the PW91 functional yields a deprotonation energy of $1176 \mathrm{~kJ} \mathrm{~mol}^{-1},{ }^{30}$ but this value does not include the aperiodic correction and should be compared with our uncorrected value, $1164 \mathrm{~kJ} \mathrm{~mol}^{-1}$. The remaining difference is small due to different basis sets and density functionals.

As we can see from Table 2, increasing the cluster size decreases the QM contribution of the deprotonation energy and simultaneously increases the total long range correction by almost the same value. This shows that the force field describes

Table 2 Deprotonation energy components $\left(\mathrm{kJ} \mathrm{mol}^{-1}\right)$ for various embedded cluster models of the $\mathrm{H}-\mathrm{CHA}$ zeolite embedded in a $2 \times 2 \times$ 2 supercell. See Table 1 for definitions

\begin{tabular}{lllllll}
\hline Model & $\Delta E_{\mathrm{DP}}^{\mathrm{LG}}$ & $E_{--} / \varepsilon(\mathrm{LG})^{a}$ & $\Delta E_{\mathrm{DP}}$ & $\Delta E_{\mathrm{DP}(\mathrm{QM})}$ & $\Delta E_{\mathrm{LR}}^{\infty} b$ & $\varepsilon(\mathrm{LG})$ \\
\hline $\mathrm{MM}$ & 1036.4 & -34.0 & 1002.3 & - & - & 3.09 \\
$8 \mathrm{~T}_{12 \mathrm{OH}}$ & 1238.0 & -37.6 & 1200.4 & 1291.7 & -53.7 & 2.79 \\
$12 \mathrm{~T}_{12 \mathrm{OH}}$ & 1239.3 & -36.1 & 1203.1 & 1276.4 & -37.1 & 2.91 \\
$16 \mathrm{~T}_{16 \mathrm{OH}}{ }^{c}$ & 1237.4 & -36.8 & 1200.7 & 1290.7 & -53.3 & 2.86 \\
$33 \mathrm{~T}_{34 \mathrm{OH}}{ }^{c}$ & 1233.5 & -24.7 & 1208.8 & 1267.3 & -33.8 & 2.83 \\
$44 \mathrm{~T}_{34 \mathrm{OH}}$ & 1232.5 & -24.0 & 1208.5 & 1267.1 & -34.6 & 2.92
\end{tabular}

${ }^{a}$ Eqn (9). ${ }^{b}$ Long range correction for LG corrected deprotonation energy, see eqn (13). ${ }^{c}$ Cluster models embedded in a $3 \times 3 \times 3$ supercell.

the electrostatic interaction consistent with the (B3LYP) functional used for its parametrization. This has been documented before for Hartree-Fock calculations with correspondingly parametrized force fields. ${ }^{18}$ Geometric parameters of the acidic site are not strongly affected by the size of the QM region, and their values converge to constant values with increasing cluster size (see ESI, $\dagger$ Table S3). These all facts prove that the applied $\mathrm{QM} / \mathrm{MM}$ methodology is appropriate to describe the properties of zeolites. Therefore, it can be used to predict the deprotonation energy of the $\mathrm{H}-2 \mathrm{dH}$ system.

\subsection{Ultra-thin $\mathrm{H}-2 \mathrm{dH}$ zeolite}

To study the acidity of the ultra-thin $\mathrm{H}-2 \mathrm{dH}$ zeolite, we performed calculations for cells of $\mathrm{H}_{n} \mathrm{Al}_{n} \mathrm{Si}_{64-n} \mathrm{O}_{128}$ with increasing $\mathrm{Al} / \mathrm{Si}$ ratio (Fig. 4), namely $1 / 63(n=1), 1 / 7(n=8)$ and $1 / 3$ $(n=16)$. The cells for the different $\mathrm{Al} / \mathrm{Si}$ ratio have the same size, but correspond to $1 \times 1,2 \times 2$, and $2 \times 2$ supercells, respectively, because the size of the primitive cells differs.

Experimentally, two-dimensional zeolites were prepared on a $\mathrm{Ru}(0001)$ substrate. ${ }^{8,9,31}$ However the interaction between zeolite and the substrate was found to be weak ${ }^{31}$ and we excluded the ruthenium layer from our computational models. We applied 2D periodic boundary conditions and calculated electrostatic interactions using the Perry summation technique, ${ }^{32,33}$ implemented in the GULP code. ${ }^{26}$ This was necessary because for standard slab calculations the energy of the charged system increases linearly with the vacuum thickness, and diverges in the limit of infinite values. ${ }^{34}$

First, we optimized the unit cell parameters using the shell model potential, which were kept fixed during all subsequent calculations. We prepared clusters of increasing size starting from a single hexagonal prism containing a Brønsted site (1D6R model) and adding up to 6 adjoining hexagonal prisms forming the 7D6R model (Fig. 5).

$\mathrm{Al} / \mathrm{Si}=1 / 631 \times 1 \mathrm{UC}$

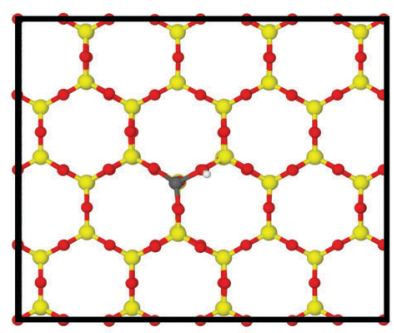

$\mathrm{Al} / \mathrm{Si}=1 / 32 \times 2 \mathrm{UC}$

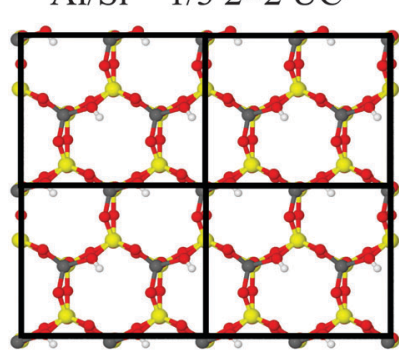

$\mathrm{Al} / \mathrm{Si}=1 / 72 \times 2 \mathrm{UC}$
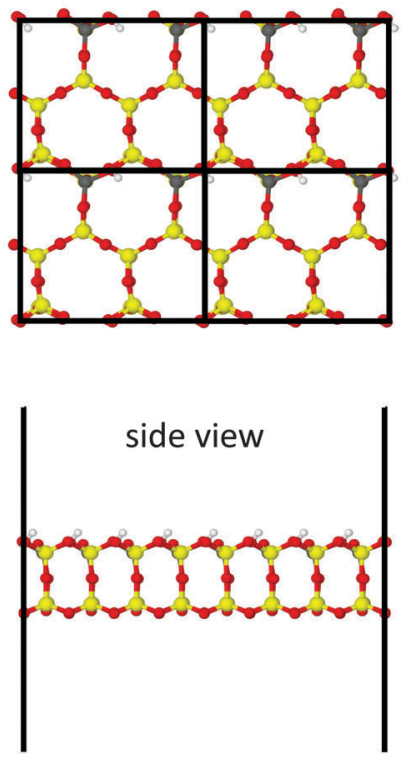

Fig. 4 Optimized cells of the $\mathrm{H}-2 \mathrm{dH}$ zeolite with different $\mathrm{Al} / \mathrm{Si}$ ratios 


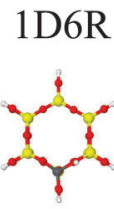

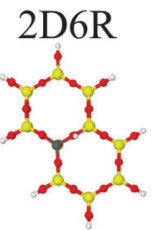
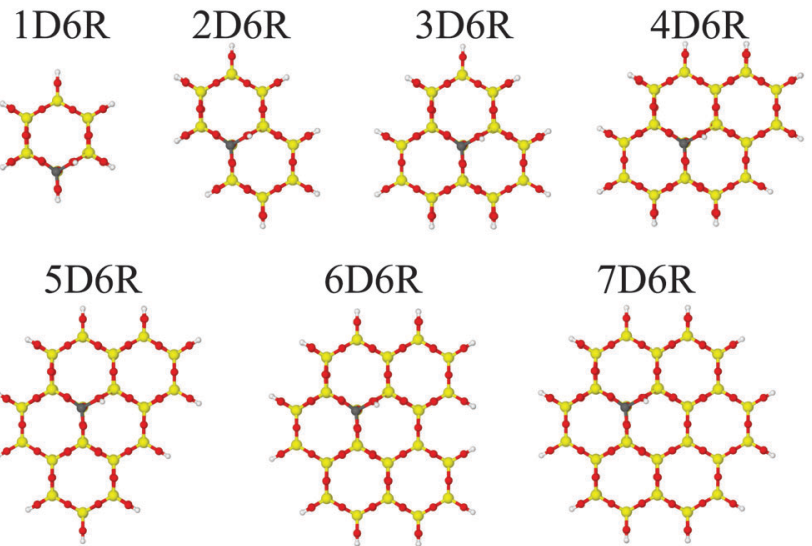

Fig. 5 Cluster models applied in QM/MM calculations of the $\mathrm{H}-2 \mathrm{dH}$ deprotonation energy $(\mathrm{Al} / \mathrm{Si}=1 / 63$, models for the other $\mathrm{Al} / \mathrm{Si}$ ratios are presented in Fig. S1 and S2 in the ESI $\dagger$ ).

In some cases it was necessary to add some extra tetrahedral sites to avoid terminal $\mathrm{O}-\mathrm{H}$ groups that are connected to neighbouring Brønsted sites. These additional T-sites are indicated by a subscript, e.g. $4 \mathrm{D} 6 \mathrm{R}_{+4 \mathrm{~T}}$ stands for four additional tetrahedral sites added to the 4DR6 cluster. Cluster models were terminated in the same way as for the H-CHA zeolite.

The simple Leslie-Gillan method of aperiodic corrections is not applicable to the $\mathrm{H}-2 \mathrm{dH}$ system. For the two-dimensional case, the macroscopic approximation is not valid anymore. To calculate corrected deprotonation energies for the $\mathrm{H}-2 \mathrm{dH}$ zeolites, we used the supercell extrapolation method which is computationally demanding. The original unit cells were multiplied in $\vec{a}$ and $\vec{b}$ directions and extrapolation curves, eqn (8), were fitted as a function of the square root of the surface area. A series of three supercells per each system, namely $1 \times 1$, $2 \times 2$, and $3 \times 3$ for $\mathrm{Al} / \mathrm{Si}=1 / 63$, and $2 \times 2,3 \times 3$, and $4 \times 4$ for $\mathrm{Al} / \mathrm{Si}=1 / 7$ and $1 / 3$, were used to extrapolate deprotonation energies. All supercell structures were optimized, both the protonated and deprotonated forms. The resulting fitting parameters and correction energies are presented in the ESI, $\dagger$ Table S4 and Fig. S3.

For each $\mathrm{Al} / \mathrm{Si}$ ratio the fitted parameters $A$ and (especially) $B$ depend on the cluster model. Nevertheless, the correction energies, $E_{\text {corr }}$ (eqn (8)), are almost constant within $6 \mathrm{~kJ} \mathrm{~mol}^{-1}$. This means that one can calculate the aperiodic correction energy using the supercell approach for only one embedded cluster model, or even at the MM level, and then apply this correction for different embedded cluster models of the same unit cell, tolerating an error of about $6 \mathrm{~kJ} \mathrm{~mol}^{-1}$.

The fitted $A$ parameters are much larger for $\mathrm{H}-2 \mathrm{dH}$ (ESI, $\dagger$ Table S4) than for H-CHA (Fig. 3). This shows that in the twodimensional $\mathrm{H}-2 \mathrm{dH}$ zeolite the interaction between charged defects is much less screened than that in the bulk $\mathrm{H}-\mathrm{CHA}$ zeolite. This is also reflected in very small effective dielectric constants of $\mathrm{H}-2 \mathrm{dH}, 1.5-1.8$ (Table 3), compared to 2.9-3.1 for H-CHA (Table 2).

The extrapolated deprotonation energies of the $\mathrm{H}-2 \mathrm{dH}$ zeolites converge to constant values with increasing embedded
Table 3 Deprotonation energy components $\left(\mathrm{kJ} \mathrm{mol}^{-1}\right)$ calculated for various embedded cluster models of $\mathrm{H}-2 \mathrm{dH}$ zeolites for different $\mathrm{Al} / \mathrm{Si}$ ratios using the supercell (SC) method

\begin{tabular}{|c|c|c|c|c|c|c|c|}
\hline $\mathrm{Al} / \mathrm{Si}$ & Model & $\Delta E_{\mathrm{DP}}^{\infty}$ & $\Delta E_{\mathrm{QM}}$ & $\Delta E_{\mathrm{LR}}^{\infty}$ & $E_{\text {rel }}$ & $E_{\text {corr(SC) }}$ & $\varepsilon_{\mathrm{eff}}(\mathrm{SC})$ \\
\hline \multirow[t]{8}{*}{$1 / 63^{a}$} & MM & 836.1 & - & - & -184.3 & -44.3 & 1.529 \\
\hline & 1D6R & 1032.1 & 1277.9 & -245.8 & -144.6 & -42.9 & 1.579 \\
\hline & 2D6R & 1037.4 & 1244.7 & -208.5 & -139.9 & -42.9 & $1.579^{b}$ \\
\hline & 3D6R & 1041.0 & 1232.6 & -182.0 & -143.2 & -45.3 & 1.493 \\
\hline & $4 \mathrm{D} 6 \mathrm{R}$ & 1041.5 & 1227.2 & -185.7 & -137.5 & -42.1 & 1.607 \\
\hline & $5 \mathrm{D} 6 \mathrm{R}$ & 1042.8 & 1217.6 & -174.9 & -137.3 & $-42.1^{d}$ & $1.607^{d}$ \\
\hline & 6D6R & 1044.8 & 1213.3 & -168.7 & -137.4 & $-42.1^{d}$ & $1.607^{d}$ \\
\hline & 7D6R & 1045.0 & 1210.1 & -165.1 & -136.9 & $-42.1^{d}$ & $1.607^{d}$ \\
\hline \multirow[t]{6}{*}{$1 / 7^{b}$} & MM & 870.8 & - & - & -191.3 & -42.5 & 1.591 \\
\hline & $1 \mathrm{D} \mathrm{R}_{+2 \mathrm{~T}}$ & 1049.3 & 1320.4 & -271.2 & -142.5 & -37.9 & 1.781 \\
\hline & $2 \mathrm{D} \mathrm{R}_{+4 \mathrm{~T}}$ & 1072.1 & 1249.2 & -177.1 & -137.2 & -44.3 & 1.523 \\
\hline & $3 \mathrm{D} 6 \mathrm{R}_{+2 \mathrm{~T}}$ & 1073.6 & 1238.3 & -164.7 & -137.8 & $-44.3^{e}$ & $1.523^{e}$ \\
\hline & 4D6R & 1068.9 & 1235.9 & -167.0 & -135.8 & -36.7 & 1.840 \\
\hline & $7 \mathrm{D} \mathrm{R}_{+4 \mathrm{~T}}$ & 1071.8 & 1229.4 & -157.6 & -134.5 & -37.9 & 1.778 \\
\hline \multirow[t]{3}{*}{$1 / 3^{c}$} & MM & 890.3 & - & - & -181.9 & -41.8 & 1.591 \\
\hline & $1 \mathrm{D} \mathrm{R}_{+4 \mathrm{~T}}$ & 1069.6 & 1290.0 & -220.4 & -143.0 & -41.3 & 1.636 \\
\hline & $4 \mathrm{D} 6 \mathrm{R}_{+4 \mathrm{~T}}$ & 1090.6 & 1241.3 & -150.7 & -138.7 & -43.0 & 1.570 \\
\hline
\end{tabular}

cluster size (Table 3 and ESI, $\dagger$ Fig. S4), which is also true for the geometrical parameters of the Brønsted sites (see ESI, $\dagger$ Table S5). The deprotonation energies of all studied $\mathrm{H}-2 \mathrm{dH}$ systems are much lower than those of H-CHA. Even the least acidic twodimensional system with $\mathrm{Al} / \mathrm{Si}=1 / 3$ has a deprotonation energy $\left(1091 \mathrm{~kJ} \mathrm{~mol}^{-1}\right.$ ) which is $142 \mathrm{~kJ} \mathrm{~mol}^{-1}$ lower than that of $\mathrm{H}-\mathrm{CHA}$ with $\mathrm{Al} / \mathrm{Si}=1 / 12$.

Table 4 compares calculated deprotonation energies and their components for $\mathrm{H}-2 \mathrm{dH}$ with different $\mathrm{Al} / \mathrm{Si}$ ratios with those for H-CHA. The table shows that the QM part of the deprotonation energy is significantly smaller for $\mathrm{H}-2 \mathrm{dH}$ than that for H-CHA. Nevertheless, the decisive factor for the very low deprotonation energy of two-dimensional zeolites is the long-range term, which (in absolute terms) is a few times larger than that for H-CHA.

The long-range component of the deprotonation energy results from the interactions between the inner part (cluster model) and its periodic surroundings. Upon removing the proton there is a change in the electrostatic (Coulomb and polarization) part of these interactions. The negative charge

Table 4 Calculated deprotonation energies and their components $\left(\mathrm{kJ} \mathrm{mol}^{-1}\right)$ for two-dimensional zeolites with different $\mathrm{Al} / \mathrm{Si}$ ratios compared to $\mathrm{H}-\mathrm{CHA}$

\begin{tabular}{|c|c|c|c|c|c|c|}
\hline System $^{a}$ & Model & $\Delta E_{\mathrm{DP}}^{\infty}$ & $\Delta E_{\mathrm{DP}(\mathrm{QM})}$ & $\Delta E_{\mathrm{LR}}^{\infty}$ & $E_{\text {rel }}$ & $\varepsilon_{\text {eff }}(\mathrm{SC})$ \\
\hline H-CHA (1/11) & $44 \mathrm{~T}_{32 \mathrm{OH}}{ }^{b}$ & 1233 & 1267 & -35 & -144 & 2.92 \\
\hline $\mathrm{H}-2 \mathrm{dH}(1 / 63)$ & $4 \mathrm{D}^{2} \mathrm{R}^{c}$ & 1042 & 1227 & -186 & -138 & 1.61 \\
\hline $\mathrm{H}-2 \mathrm{dH}(1 / 7)$ & $4 \mathrm{D} 6 \mathrm{R}^{d}$ & 1069 & 1236 & -167 & -136 & 1.84 \\
\hline $\mathrm{H}-2 \mathrm{dH}(1 / 3)$ & $4 \mathrm{D} 6 \mathrm{R}_{+4 \mathrm{~T}}{ }^{c}$ & 1091 & 1241 & -151 & -139 & 1.57 \\
\hline
\end{tabular}

${ }^{a} \mathrm{Al} / \mathrm{Si}$ ratio is in parentheses. ${ }^{b} 3 \times 3 \times 3$ unit cell. $^{c} 2 \times 2$ unit cell. d $4 \times 4$ unit cell. 
created upon deprotonation is stabilized by its surroundings. This stabilization is maximum if there is no screening of the created charge $(\varepsilon=1)$. The more efficient the screening (the larger $\varepsilon$ ), the smaller is the electrostatic stabilization of the created charge. For the $\mathrm{H}-2 \mathrm{dH}$ zeolites the dielectric constant is relatively small, therefore the created charge is more stabilized by the crystal field than the one in H-CHA. If this interpretation is correct, one should observe a dependence of the deprotonation energy of zeolites on their dielectric constant.

The geometric parameters of the Brønsted sites of $\mathrm{H}-2 \mathrm{dH}$ $(\mathrm{Al} / \mathrm{Si}=1 / 63)$ and $\mathrm{H}-\mathrm{CHA}$ are almost the same (see ESI, $\dagger$ Tables $\mathrm{S} 1$ and S2) and the relaxation energies, $E_{\text {rel }}$, of two-dimensional and bulk zeolites (Table 4) are also very similar. Both these facts confirm that differences in long range electrostatic interactions are indeed responsible for the observed differences in deprotonation energies.

\subsection{Effect of the $\mathrm{Al} / \mathrm{Si}$ ratio}

The deprotonation energy of the $\mathrm{H}-2 \mathrm{dH}$ zeolites increases with increasing $\mathrm{Al} / \mathrm{Si}$ ratio (Table 4 and Fig. $\mathrm{S} 4$ in the ESI $\dagger$ ). This behaviour was found in a number of experimental ${ }^{12,35,36}$ and theoretical studies. ${ }^{37-39}$ It suggests that an important factor governing the acidity of the Brønsted site is its local environment, i.e. the number of $\mathrm{AlO}_{4}$ tetrahedra in the second coordination sphere of the particular acidic site. ${ }^{12,35-38,40,41}$ An increase in the $\mathrm{Al} / \mathrm{Si}$ ratio also affects the structure of the protonated and the deprotonated Brønsted sites (Table S5 in the ESI $\dagger$ ), namely it decreases the $\mathrm{Al}-\mathrm{O}$ bond length and the $\mathrm{Al}-\mathrm{O}-\mathrm{Si}$ angle and increases the $\mathrm{Si}-\mathrm{O}$ bond length. Geometric parameters do not affect the relaxation energy of the anion, which is independent of the $\mathrm{Al} / \mathrm{Si}$ ratio (Table 4). This suggests that the decrease of the acidity with increase of the Al content is caused mainly by the changes of the electronic structure of the zeolite. In 3D zeolites this effect is localised, ${ }^{37,38}$ namely it is caused by the interaction of the Brønsted site with its nearest surroundings. However, comparison of the QM and long range contributions of the deprotonation energies of $\mathrm{H}-2 \mathrm{dH}$ zeolites (Table 4) shows that the increase of the $\mathrm{Al} / \mathrm{Si}$ ratio also affects the long range term.

\subsection{Dielectric screening in two-dimensional systems}

The small dielectric constant of the $\mathrm{H}-2 \mathrm{dH}$ thin film can be explained by classical electrostatics, using the images. ${ }^{42}$ Let us first consider an electrostatic potential generated by a point charge $q$ immersed in a homogeneous dielectric film, characterised by a dielectric constant $\varepsilon_{1}$ and the thickness $L$, surrounded by a medium with a dielectric constant $\varepsilon_{2}$ (Fig. 6 with $\varepsilon_{3}=\varepsilon_{2}$ ).

The charge placed in the point $(0,0, a)$ generates the following potential (in atomic units) at the point $(x, 0, a):{ }^{43}$

$$
V_{2 \mathrm{D}}(x, a, L)=\frac{q}{\varepsilon_{1}} \sum_{n=-\infty}^{n=\infty} \frac{\gamma^{|n|}}{\sqrt{x^{2}+\left(\frac{L}{2}-a-a_{n}\right)^{2}}}
$$

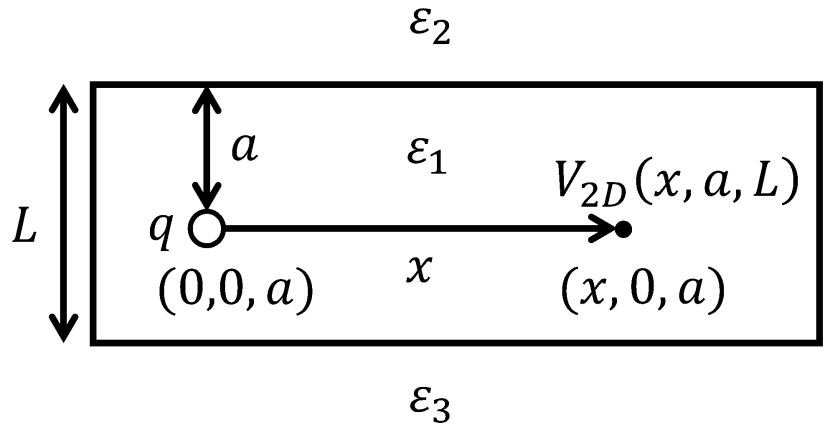

Fig. 6 Model for dielectric constant calculations.

With

$$
\gamma=\frac{\left(\varepsilon_{1}-\varepsilon_{2}\right)}{\left(\varepsilon_{1}+\varepsilon_{2}\right)}
$$

$$
a_{n}=n L+(-1)^{n}\left(\frac{L}{2}-a\right)
$$

Let us now consider an electrostatic potential generated by the point charge $q$ immersed in the homogenous dielectric medium of a bulk system, characterized by the dielectric constant $\varepsilon$. At a distance $x$ from the charge the potential (in atomic units) is:

$$
V_{3 \mathrm{D}}(x)=\frac{q}{\varepsilon x}
$$

By comparison of eqn (16a) and (17) we can determine the effective dielectric constant $\varepsilon_{\text {eff }}=\varepsilon$ at the point $(x, 0, a)$ of the dielectric slab:

$$
\varepsilon_{\mathrm{eff}}(x, a, L)=\frac{\varepsilon_{1}}{x}\left(\sum_{n=-\infty}^{n=\infty} \frac{\gamma^{|n|}}{\sqrt{x^{2}+\left(\frac{L}{2}-a-a_{n}\right)^{2}}}\right)^{-1}
$$

In contrast to bulk dielectrics, the effective dielectric constant of thin layers depends on the distance from the charge (Fig. 7). Independent of the sample's thickness, the effective dielectric constant on the surface $(a=0)$ changes continuously from $\left(\varepsilon_{1}+\right.$ $\left.\varepsilon_{2}\right) / 2$ near the charge to $\varepsilon_{2}$ at an infinite distance from it (Fig. 7). In the case of the $\mathrm{H}-2 \mathrm{dH}$ thin film, for which $\varepsilon_{1}$ (calculated as $1 / 3$ of the trace of the dielectric constant tensor, which was determined from the shell-model potential calculations) is about 5 and $\varepsilon_{2}$ is equal to 1 (vacuum), the theoretical dielectric constant should decrease from 3 near the charge to 1 at large distances. During derivation of eqn (18) a few assumptions were made, among others the assumption of homogenous dielectric medium and the assumption that the border between two dielectric media is sharp. Nevertheless, the calculated dielectric constant (1.6-1.9, Table 4) falls in the theoretical range $(1<\varepsilon<3)$, but a precise theoretical value cannot be specified.

We can use eqn (18) to predict the qualitative behaviour of zeolite dielectric properties. For example, we know that the effective dielectric constant depends on the distance $a$ from the 

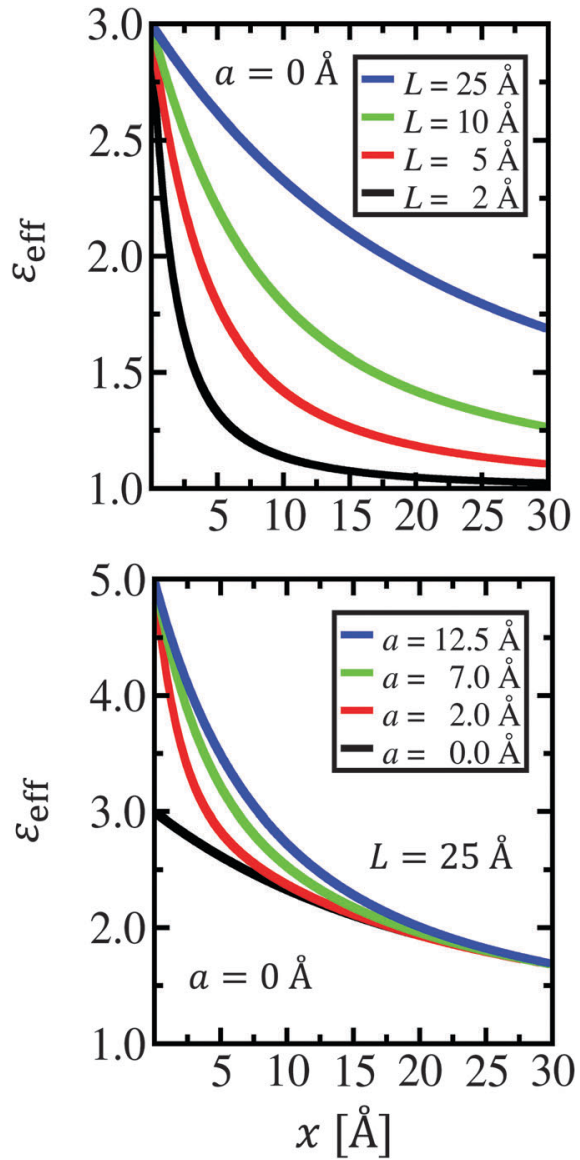

Fig. 7 Theoretical effective dielectric constant (eqn (18)) as a function of a distance $x$ from the charge calculated for $\varepsilon_{1}=5.0$ and $\varepsilon_{2}=1.0$ and different $a$ and $L$ parameters.

surface (Fig. 7). In other words, when $\varepsilon_{1}>\varepsilon_{2}$, increasing $a$ increases the dielectric constant. This is an important statement because it means that in the case of two-dimensional zeolites we cannot compare uncorrected deprotonation energies of two different Brønsted sites which are at different distances from the surface, even though the size of the unit cell is the same. This also suggests that the acidity of the Brønsted sites which are deeper in the sample can be lower than of those which are at the surface. The thickness of the sample changes the slope of the dielectric constant curve, namely with decrease of $L$ the curve becomes steeper (Fig. 7). Hence, we can expect that the acidity of the surface Brønsted sites should increase with decrease of the sample thickness. To confirm these predictions, a separate study of the thickness dependence of deprotonation energies for thin H-MFI films ${ }^{6}$ is underway.

Let us consider the most general situation, namely a thin film characterized by a macroscopic dielectric constant $\varepsilon_{1}$ surrounded by different media at the top and the bottom with dielectric constants $\varepsilon_{2}$ and $\varepsilon_{3}$, respectively. This corresponds to our experimental model system, namely the ultrathin zeolite film deposited on the metal surface $\left(\varepsilon_{3}=\infty\right)$ under ultra-high vacuum conditions $\left(\varepsilon_{2}=1.0\right)$. Once again we can apply the macroscopic approximation to calculate the electrostatic potential

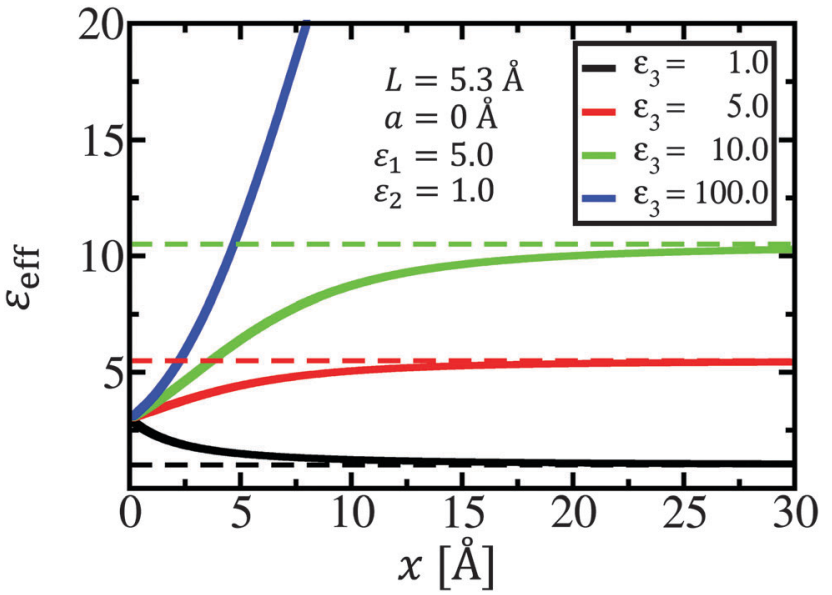

Fig. 8 Theoretical effective dielectric constant as a function of a distance from the charge calculated for $\varepsilon_{1}=5.0, \varepsilon_{2}=1.0, L=5.3 \AA, a=0.0 \AA$ and different dielectric constants of the bottom layer $\left(\varepsilon_{3}\right)$.

within a film and subsequently the effective dielectric constant of the film. ${ }^{43}$ Fig. 8 shows the effective dielectric constant within the thin layer as a function of a distance $x$ from the point charge, calculated for different dielectric constants of the bottom layer. The effective dielectric constant on the slab surface $(a=0)$ changes continuously from $\left(\varepsilon_{1}+\varepsilon_{2}\right) / 2$ near the charge to $\left(\varepsilon_{2}+\varepsilon_{3}\right) / 2$ at large distances. It means that in the experimental case the dielectric screening near the charge is smaller than in the bulk zeolite, but for the bigger distances it quickly approaches infinity. Therefore the long range interaction between the active site and the crystal lattice should be much reduced with respect to the system without the metal substrate. If we assume that the influence of the metal substrate on the QM part of the deprotonation energy is not strong (short range interaction) we can expect that the acidity of the ultra-thin zeolite adsorbed on the metallic surface should be reduced with respect to the free-standing film.

\subsection{Comparison with $\mathrm{OH}$ frequency shifts}

Since deprotonation energies cannot be directly measured for solid acids, a linear relationship has been suggested (Paukshtis and Yurchenko $)^{44,45}$ between the $\mathrm{OH}$ frequency shift upon complex formation with a weak base molecule and the enthalpy of deprotonation, $\Delta H_{\mathrm{DP}}\left(\mathrm{kJ} \mathrm{mol}^{-1}\right)$ :

$$
\Delta H_{\mathrm{DP}}=1390-442.5 \cdot \log \left(\Delta \tilde{\nu}_{\mathrm{OH}} / \Delta \tilde{\nu}_{\mathrm{SiOH}}\right)
$$

Here, $\Delta \tilde{\nu}_{\mathrm{OH}}$ and $\Delta \tilde{\nu}_{\mathrm{SiOH}}$ are the wavenumber shifts for the O-H stretching vibrations of Brønsted sites and free silanols, respectively (in $\mathrm{cm}^{-1}$ ), caused by the adsorption of weak bases. Because we are interested in the difference between two different zeolites, $\Delta\left(\Delta E_{\mathrm{DP}}\right)$, we do not need to know $\Delta \tilde{\nu}_{\mathrm{SiOH}}$, which cancels out:

$$
\Delta\left(\Delta E_{\mathrm{DP}}\right) \approx \Delta\left(\Delta H_{\mathrm{DP}}\right)=-442.5 \cdot \log \left(\Delta \tilde{\nu}_{\mathrm{OH}(\mathrm{H}-2 \mathrm{dH})} / \Delta \tilde{\nu}_{\mathrm{OH}(\mathrm{H}-\mathrm{CHA})}\right)
$$

The wavenumber shifts upon CO adsorption from ref. 9, which have been calculated for exactly the same $\mathrm{Si} / \mathrm{Al}=7$ bilayer as examined in the present study, yield a deprotonation energy 
difference of only $12 \mathrm{~kJ} \mathrm{~mol}^{-1}$, one order of magnitude less than the calculated difference of $164 \mathrm{~kJ} \mathrm{~mol}^{-1}$ from Table 4 . Insertion of experimental values of $\Delta \tilde{\nu}_{\mathrm{OH}(\mathrm{H}-2 \mathrm{dH})}$ and $\Delta \tilde{\nu}_{\mathrm{OH}(\mathrm{H}-\mathrm{CHA})}, 379$ and $316 \mathrm{~cm}^{-1}$, respectively, ${ }^{9}$ into eqn (20) yields a larger deprotonation energy difference of $35 \mathrm{~kJ} \mathrm{~mol}^{-1}$, but which is still much smaller than the calculated value of $164 \mathrm{~kJ} \mathrm{~mol}^{-1}$.

The obvious reason for the breakdown of the $\Delta H_{\mathrm{DP}}-\Delta \tilde{\nu}_{\mathrm{OH}}$ plot is that the $\mathrm{OH}$ stretching frequency shift upon adsorption of CO refers to an "early" stage of the reaction, whereas deprotonation refers to a "late" stage of the catalytic reaction when the proton has been removed. This is consistent with the finding that the much larger acidity "measured" with the deprotonation energy is largely due to the better stabilization of the created negative charge in media with low dielectric constants such as two-dimensional zeolites.

\section{Conclusions}

$\mathrm{QM} / \mathrm{MM}$ calculations of deprotonation energies of ultrathin $\mathrm{H}-2 \mathrm{dH}$ zeolites and the bulk H-CHA zeolite confirm the much higher acidity of the ultra-thin zeolites compared to bulk systems, previously observed in experiments. ${ }^{8,9}$ The very low deprotonation energy of the $\mathrm{H}-2 \mathrm{dH}$ system reflects the fact that the dielectric constant of thin dielectrics immersed in a vacuum is much lower than that of corresponding bulk systems, resulting in a better stabilization of the charge created upon deprotonation. The dielectric constant of a thin film depends on its thickness and on the distance of the charge from the surface. This suggests that the properties of acidic sites in ultra-thin zeolites ${ }^{6,7}$ and single layer zeolites ${ }^{8,9}$ vary with their distance from the surface and can be tuned by changing the sample's thickness. Another consequence is that the acidity of two-dimensional systems as judged upon deprotonation energies is much larger than $\mathrm{OH}$ frequency shifts upon adsorption of base molecules indicated.

\section{Acknowledgements}

This work has been supported by the German Research Foundation (DFG) through Collaborative Research Center Grant CRC 1109. The authors would like to thank Milan Ončák and Radosław Włodarczyk for helpful discussions.

\section{References}

1 A. Corma, Chem. Rev., 1995, 95, 559.

2 A. Corma, J. Catal., 2003, 216, 298.

3 W. O. Haag, R. M. Lago and P. B. Weisz, Nature, 1984, 309, 589. 4 W. J. Mortier, J. Sauer, J. A. Lercher and H. Noller, J. Phys. Chem., 1984, 88, 905.

5 W. J. Roth, P. Nachtigall, R. E. Morris and J. Čejka, Chem. Rev., 2014, 114, 4807.

6 M. Choi, K. Na, J. Kim, Y. Sakamoto, O. Terasaki and R. Ryoo, Nature, 2009, 461, 246.

7 Y. Seo, K. Cho, Y. Jung and R. Ryoo, ACS Catal., 2013, 3, 713.
8 J. A. Boscoboinik, X. Yu, B. Yang, F. D. Fischer, R. Włodarczyk, M. Sierka, S. Shaikhutdinov, J. Sauer and H.-J. Freund, Angew. Chem., Int. Ed., 2012, 51, 6005.

9 J. A. Boscoboinik, X. Yu, E. Emmez, B. Yang, S. Shaikhutdinov, F. D. Fischer, J. Sauer and H.-J. Freund, J. Phys. Chem. C, 2013, 117, 13547.

10 A. Zecchina, G. Spoto and S. Bordiga, Phys. Chem. Chem. Phys., 2005, 7, 1627.

11 J. Sauer, J. Mol. Catal., 1989, 54, 312.

12 W. E. Farneth and R. J. Gorte, Chem. Rev., 1995, 95, 615.

13 E. G. Derouane, J. C. Védrine, R. R. Pinto, P. M. Borges, L. Costa, M. A. N. D. A. Lemos, F. Lemos and F. R. Ribeiro, Catal. Rev., 2013, 55, 454.

14 J. E. Bartmess, J. A. Scott and R. T. McIver, Jr., J. Am. Chem. Soc., 1979, 101, 6046.

15 H. V. Brand, L. A. Curtiss and L. E. Iton, J. Phys. Chem., 1993, 97, 12773.

16 M. Brändle and J. Sauer, J. Am. Chem. Soc., 1998, 120, 1556. 17 M. Leslie and M. J. Gillan, J. Phys. C: Solid State Phys., 1985, 18, 973.

18 U. Eichler, M. Brändle and J. Sauer, J. Phys. Chem. B, 1997, 101, 10035.

19 U. Eichler, C. Kölmel and J. Sauer, J. Comput. Chem., 1997, 18, 463.

20 M. Sierka and J. Sauer, J. Chem. Phys., 2000, 112, 6983.

21 M. Sierka and J. Sauer, Faraday Discuss., 1997, 106, 41.

22 TURBOMOLE V6.4; a development of the University of Karlsruhe and Forschungszentrum Karlsruhe $\mathrm{GmbH}$, http:/www.turbomole.com, 2012.

23 F. Weigend, M. Häser, H. Patzelt and R. Ahlrichs, Chem. Phys. Lett., 1998, 294, 143.

24 F. Weigend and R. Ahlrichs, Phys. Chem. Chem. Phys., 2005, 7, 3297.

25 M. Sierka and J. Sauer, J. Phys. Chem. B, 2001, 105, 1603.

26 J. D. Gale, J. Chem. Soc., Faraday Trans., 1997, 93, 629.

27 Jmol: an open-source Java viewer for chemical structures in 3D, http://www.jmol.org/.

28 M. Calligaris, G. Nardin, L. Randaccio and P. C. Chiaramonti, Acta Crystallogr., 1982, 38, 602.

29 J. Sauer, K.-P. Schröder and V. Termath, Collect. Czech. Chem. Commun., 1998, 63, 1394.

30 C. Lo and B. L. Trout, J. Catal., 2004, 227, 77.

31 R. Włodarczyk, M. Sierka, J. Sauer, D. Löffler, J. J. Uhlrich, X. Yu, B. Yang, I. M. N. Groot, S. Shaikhutdinov and H. J. Freund, Phys. Rev. B: Condens. Matter Mater. Phys., 2012, 85, 085403.

32 D. E. Parry, Surf. Sci., 1975, 49, 433.

33 D. E. Parry, Surf. Sci., 1976, 54, 195.

34 E. Wasserman, J. R. Rustad and A. R. Felmy, Surf. Sci., 1999, 424, 19.

35 H. Stach, J. Jaenchen, H. G. Jerschkewitz, U. Lohse, B. Parlitz and M. Hunger, J. Phys. Chem., 1992, 96, 8480.

36 B. Gil, E. Broclawik, J. Datka and J. Klinowski, J. Phys. Chem., 1994, 98, 930.

37 L. Grajciar, C. O. Arean, A. Pulido and P. Nachtigall, Phys. Chem. Chem. Phys., 2010, 12, 1497. 
38 M. Sierka, U. Eichler, J. Datka and J. Sauer, J. Phys. Chem. B, 1998, 102, 6397.

39 A. G. Pel'menshchikov, E. A. Paukshtis, V. G. Stepanov, V. I. Pavlov, E. N. Yurchenko, K. G. Ione, G. M. Zhidomirov and S. Beran, J. Phys. Chem., 1989, 93, 6725.

40 G. M. Zhidomirov and V. B. Kazansky, in Advances in Catalysis, ed. D. D. Eley, H. Pines and P. B. Weisz, Academic Press, 1986, vol. 34, p. 131.
41 J. Datka and B. Gil, J. Catal., 1994, 145, 372.

42 J. Jackson, Classical Electrodynamics, Wiley, 3rd edn, 1998.

43 M. Kumagai and T. Takagahara, Phys. Rev. B: Condens. Matter Mater. Phys., 1989, 40, 12359.

44 E. A. Paukshtis, R. I. Soltanov and E. N. Yurchenko, React. Kinet. Catal. Lett., 1981, 16, 93.

45 A. P. Evgenii and E. N. Yurchenko, Russ. Chem. Rev., 1983, 52, 242. 